\title{
Les âges absolus en Sciences de la Terre
}

\author{
Jacqueline VANDER AUWERA \\ Département de Géologie, Université de Liège, B-4000 Sart Tilman
}

\section{Résumé}

La stratigraphie et la paléontologie ont été très rapidement utilisées par les géologues pour placer leurs observations dans un cadre temporel. Néanmoins, la découverte de la radioactivité et l'application des techniques de physique nucléaire aux Sciences de la Terre a permis d'obtenir des âges absolus. C'est ainsi que l'âge de la Terre a pu être déterminé en datant les météorites et qu'on a pu détecter la présence de terrains très anciens à la surface de la Terre.

Mots clés : Géologie - Radioactivité - Âge de la Terre

\begin{abstract}
Very early, stratigraphy and paleontology have been used by geologists to place time constraints on their observations. However, the discovery of radioactivity and the application of nuclear physics techniques to the Earth Sciences have enabled the determination of absolute ages. It was thus possible to determine the age of the Earth by using meteorites and to detect the presence of very old formations (Hadean) at the surface of the Earth.
\end{abstract}

Keywords: Geology - Radioactivity - Age of the Earth

\section{Introduction}

Comme les processus géologiques se déroulent sur des intervalles de temps qui sont le plus souvent très longs, les géologues sont continuellement confrontés à la variable temps. C'est ainsi qu'une échelle des temps géologiques a été conçue et subdivisée en différentes unités appelées successivement éons, ères, périodes en fonction de l'intervalle de temps considéré. Les formations géologiques observées sur Terre dont l'âge a pu être déterminé peuvent ainsi être placées dans un cadre temporel. Le dépôt de sédiments dans les lacs, les rivières et les océans produit des couches qui s'empilent les unes sur les autres pour finalement donner des strates dont la plus ancienne est la première déposée. Ce principe simple est à la base de la stratigraphie qui a permis de débrouiller les enregistrements sédimentaires préservés dans les terrains anciens. Par ailleurs, les différences observées par les paléontologues entre les restes fossiles d'anciennes espèces de faunes et de flores ont permis d'établir une échelle de temps découpée en intervalles paléontologiques caractérisés 
par des fossiles connus. Ce n'est qu'avec la découverte de la radioactivité et le développement d'instruments capables de mesurer avec suffisamment de précision les rapports isotopiques des éléments que les géologues ont eu accès à des âges absolus qui ont notamment permis de dater ces assemblages de fossiles.

\section{La radioactivité et l'équation de l'isochrone}

Le processus de la radioactivité a été découvert par $\mathrm{H}$. Becquerel ainsi que P. et M. Curie pendant la période de 1896 à 1902. La radioactivité permet à des nuclides instables de se désintégrer pour produire un nouveau nuclide qui sera lui-même aussi instable ou stable. Ce processus suit une loi simple (loi Curie-Rutherford-Soddy) (Rutherford et Soddy, 1902) :

$$
\frac{d N}{d t}=\quad N
$$

où $N$ est le nombre de nuclides, $\lambda$ est la constante de désintégration radioactive du nuclide considéré et t est le temps. L'intégration de cette loi donne :

$$
N=N_{0} \mathrm{e}^{-\lambda t}
$$

où $N$ est le nombre de nuclides restant à l'heure actuelle et $N_{0}$ étant le nombre de nuclides présents lors de la formation du système. Les différents types de radioactivité, $\beta^{-}, \beta^{+}$, capture électronique, $\alpha$, fission spontanée suivent cette même loi de la radioactivité. Une des difficultés de cette équation est de déterminer $N_{0}$. On utilise pour cela l'isotope fille $(D)$ qui est produit par la désintégration de $N$ et on obtient l'équation suivante :

$$
D=D_{0}+N\left(\mathrm{e}^{\lambda t}-1\right)
$$

où $D$ est la quantité de l'isotope fille mesuré maintenant et $D_{0}$, l'isotope fille qu'il y avait dans le système au moment de sa formation. Cette équation est souvent appelée l'équation de l'isochrone et dans les cas favorables elle permet la détermination simultanée de t, l'âge de l'événement géologique étudié, et $\mathrm{D}_{0}$. 
Bulletin de la Société Royale des Sciences de Liège,

Vol. 85, 2016, Actes de colloques, Le temps , p. 17 - 20

\section{L'âge de la Terre}

La détermination de l'âge de la Terre a fait l'objet d'une longue controverse qui a opposé les physiciens et les géologues. En effet, pour Newton et Descartes, la Terre ne pouvait avoir que quelques milliers d'années. Cette estimation était en contradiction avec les déductions faites par les premiers géologues qui sur base de leurs observations de terrain et de la durée estimée des processus géologiques pensaient que l'âge de la Terre devait être beaucoup plus élevé. Plus tard, lord Kelvin a calculé la durée du refroidissement de la Terre en se basant sur des mesures du flux de chaleur et a conclu que la Terre devait avoir entre 40 et 100 millions d'années. Cet âge était encore bien trop court par rapport aux estimations des géologues, notamment Lyell. Cette différence résultait du fait qu'à l'époque de Kelvin la radioactivité n'était pas connue et on ignorait que la désintégration radioactive des éléments tels que l'U, le Th et le $\mathrm{K}$ qui sont présents dans les formations géologiques provoque un dégagement de chaleur qui n'avait pas pu être pris en compte dans les calculs de Kelvin. L'histoire scientifique donnera plus tard définitivement raison aux géologues puisque l'âge de la Terre est maintenant bien contraint à 4.55 milliards d'années.

La Terre étant une planète «vivante », les formations géologiques et les roches ont souvent une histoire longue et complexe avec plusieurs événements de déformations, de métamorphisme et de magmatisme. Il n'est donc pas possible de déterminer l'âge de la Terre avec ce type d'échantillons. Les géologues se sont donc tournés vers les météorites pour quantifier l'âge de la Terre. En effet, les météorites viennent d'autres parties du système solaire, la plupart de la ceinture des astéroïdes qui sépare les planètes telluriques des planètes géantes, et se sont presque toutes formées lors de la naissance du système solaire. Il y a deux grands types de météorites, les météorites différenciées et non différenciées. Les premières ont été extraites de planétésimaux qui ont subi une étape de différenciation primordiale et possèdent plusieurs couches de compositions distinctes. Les secondes ont une composition primitive. C'est le cas en particulier de la catégorie des chondrites carbonées qui ont été reconnues comme les corps les plus primitifs. Elles ont été datées à l'aide de plusieurs systèmes isotopiques et tous les âges obtenus convergent vers 4.55 Ga (Faure, 1986).

\section{Les formations géologiques les plus anciennes}

La plus ancienne période de la Terre s'appelle l'Hadéen et va de 4.55 à $4.03 \mathrm{Ga}$. Il reste très peu de traces de cette époque de la Terre probablement parce que la convection mantellique et la tectonique des plaques ont presque complètement effacé ces formations. Ces reliques comprennent: les complexes de gneiss d'Acasta (Canada) (4.03 Ga), d'Itsaq (Groenland) (3.85-3.60 Ga), du Napier (Antarctique) (3.95-3.8 Ga), le bloc Saglek-Hebron (Labrador, Canada) (3.95 Ga), la chaîne supracrustale Nuvvuagittuq (Canada) (3.75Ga) et la zone Anshan (Chine) (3.8 Ga). La plupart de ces formations anciennes sont principalement constituées de roches de composition felsique (riche en feldspaths et quartz) faites de suites 
dites TTG (Tonalite-Trondhjemite-Granite) ainsi que des roches ultramafiques et métasédimentaires mineures (Bowring and Williams, 1999).

Pour dater ces formations anciennes, les géologues utilisent le zircon $\left(\mathrm{ZrSiO}_{4}\right)$, minéral assez ubiquiste et qui a la propriété d'être résistant aux phénomènes d'altération et aux différents événements métamorphiques qui ont pu affecter ces roches. L'âge de formation de ce minéral peut être obtenu en utilisant le système isotopique U-Pb et la méthode de la courbe Concordia. En effet, $1^{1} \mathrm{U}^{4+}$,isotope parent de ce système, a un rayon ionique voisin de celui du $\mathrm{Zr}^{4+}$ et pourra donc facilement s'incorporer dans la structure de ce minéral. Par contre, le $\mathrm{Pb}^{2+}$, isotope fille de ce système, a une charge plus petite et un rayon ionique nettement plus grand. Il entre donc très difficilement dans la structure du zircon et on peut donc en première approximation négliger la quantité initiale de $\mathrm{Pb}\left(\mathrm{Pb}_{0}\right)$.

La formation de Jack Hills située dans la partie occidentale de l'Australie à $800 \mathrm{~km} \mathrm{au}$ Nord de la ville de Perth est constituée de formations sédimentaires détritiques qui résultent de l'érosion de lambeaux de croûte continentale. Les sédiments ainsi produits ont ensuite été déposés et puis transformés en roches par la diagenèse. Dans ces formations, les géologues ont observés des grains de zircons et la datation de l'un d'entre eux a donné l'âge le plus ancien obtenu sur Terre : $4.4 \mathrm{Ga}$ (Wilde et al., 2001). Ce résultat remarquable montre qu'il y a eu très tôt après l'accrétion de notre planète, une croûte continentale froide.

\section{Références}

Bowring, S.A., Williams, I.S. (1999). Priscoan (4.00-4.03 Ga) orthogneisses from northwestern Canada. Contrib. Mineral. Petrol., 134, 3-16.

Faure, G. (1986). Principles of isotope geology. Wiley ans Sons, 589p.

Rutherford, E., Soddy, F. (1902). The radioactivity of thorium compounds II. The cause and nature of radioactivity. J. Chem. Soc. London, 81, 837-60.

Wilde,S.A., Valley, J.W., Peck, W.H., Graham, C.M. 2001. Evidence from detrital zircons for the existence of continental crust and oceans on the Earth 4.4 Gyr ago. Nature, 409, 175-178. 